OPEN ACCESS

Edited by:

Yanmei Tie,

Brigham and Women's Hospital and Harvard Medical School,

United States

Reviewed by:

Andrei Holodny,

Memorial Sloan Kettering Cancer

Center, United States

Marco Riva,

University of Milan, Italy

Lei Jin,

Fudan University, China

*Correspondence:

Zhentao Zuo

ztzuo@bcslab.ibp.ac.cn

Songbai Gui

guisongbai@yeah.net

Specialty section:

This article was submitted to

Cancer Imaging and Image-directed Interventions,

a section of the journal

Frontiers in Oncology

Received: 14 October 2020 Accepted: 12 January 2021

Published: 26 February 2021

Citation:

Jin L, Li C, Zhang Y,

Yuan T, Ying J, Zuo Z and Gui S (2021) The Functional Reorganization

of Language Network Modules in Glioma Patients: New Insights From Resting State fMRI Study.

Front. Oncol. 11:617179. doi: 10.3389/fonc.2021.617179

\section{The Functional Reorganization of Language Network Modules in Glioma Patients: New Insights From Resting State fMRI Study}

\author{
Lu Jin ${ }^{1}$, Chuzhong Li ${ }^{2}$, Yazhuo Zhang ${ }^{2}$, Taoyang Yuan ${ }^{2}$, Jianyou Ying ${ }^{2}$, Zhentao Zuo $^{3 *}$ \\ and Songbai Gui ${ }^{\text {* }}$ \\ 1 Department of Neurosurgery, Beijing Tiantan Hospital, Capital Medical University, Beijing, China, ${ }^{2}$ Beijing Neurosurgical \\ Institute, Capital Medical University, Beijing, China, ${ }^{3}$ State Key Laboratory of Brain and Cognitive Science, Institute of \\ Biophysics, Chinese Academy of Sciences, Beijing, China
}

Background: Prior investigations of language functions have focused on the response profiles of particular brain regions. However, the specialized and static view of language processing does not explain numerous observations of functional recovery following brain surgery. To investigate the dynamic alterations of functional connectivity (FC) within language network $(\mathrm{LN})$ in glioma patients, we explored a new flexible model based on the neuroscientific hypothesis of core-periphery organization in LN.

Methods: Group-level LN mapping was determined from 109 glioma patients and fortytwo healthy controls (HCs) using independent component analysis (ICA). FC and mean network connectivity ( $\mathrm{mNC}$ : I/rFCW, FCb, and FCg) were compared between patients and HCs. Correlations between mNC and tumor volume (TV) were calculated.

Results: We identified ten separate LN modules from ICA. Compared to HCs, glioma patients showed a significant reduction in language network functional connectivity (LNFC), with a distinct pattern modulated by tumor position. Left hemisphere gliomas had a broader impact on FC than right hemisphere gliomas, with more reduced edges away from tumor sites $(p=0.011)$. $\mathrm{mNC}$ analysis revealed a significant reduction in all indicators of FC except for IFCW in right hemisphere gliomas. These alterations were associated with TV in a double correlative relationship depending on the tumor position across hemispheres.

Conclusion: Our findings emphasize the importance of considering the modulatory effects of core-periphery mechanisms from a network perspective. Preoperative evaluation of changes in LN caused by gliomas could provide the surgeon a reference to optimize resection while maintaining functional balance.

Keywords: language network, mean functional network connectivity, glioma, surgery, reorganization 


\section{INTRODUCTION}

Traditionally, the posterior part of the left superior temporal gyrus (Wernicke's area) and the rostral part of the left inferior frontal cortices (Broca's area) have been associated with language comprehension and production and are classically designated as "eloquent" areas $(1,2)$. However, recent studies have revealed that additionally bilateral temporal, parietal, prefrontal, and putamen regions (3) and even the cerebellum (4) are involved in language processing, reflecting a large-scale network engaging in language comprehension and production distributed at both the cortical and subcortical levels. Within this distributed language processing system, the left hemisphere frontotemporal subnetwork is widely assumed to underpin language comprehension in the key combinatorial language domains of grammatical computations and semantic operations (5), while the right hemisphere subnetwork contributes to linguistic working memory capacity (6, 7). Together, these findings indicate that the language system is best considered a core-periphery model regulated by a domain-general and domain-specific subnetwork (8), an approach that has been proven to be constructive in understanding the functional architecture of language (9).

As the language network strikes a balance between integration and segregation among functionally specialized brain regions, we hypothesized that distinct structural damage would lead to different maladaptation and reorganization of inter versus intra-hemisphere connectivity due to their distinct roles in the network. Compared with task-based fMRI, restingstate fMRI obviates task compliance from patients, allows the parallel assessment of functional networks. Abnormal communications within brain functional network has been reported in series of brain lesion studies using resting-state fMRI functional connectivity $(10,11)$, however, the degree to which lesion topography (sizes, locations) accounts for the variability of functional connectivity across different modules is mostly unknown.

Thus, we subdivided the language-related regions into four subsystems, the left frontal module, the left temporal module, and their contralateral homologues (12). Then, we performed ICA to identify LN maps at rest in the presence of structural brain damage caused by gliomas located in the four subsystems, and compared FC and mNC among LN modules between glioma patients and demographically matched HCs. Finally, the possible correlations between TV and $\mathrm{mNC}$ patterns in glioma patients were assessed.

\section{MATERIALS AND METHODS}

\section{Participants}

112 glioma patients were recruited from our Department of Neurosurgery, and forty-two demographically matched HCs were included in this study. These patients were selected from a pool of database collected between December 2016 November 2017. The patient selection flowchart can be seen in Supplementary Figure S1. All participants met the following criteria (1): Patients with a glioma affecting one of the language subsystem areas; (2) No symptoms of motor impairment; (3) Right hand according to the Edinburgh Inventory (13); (4) No history of brain surgery and psychiatric illness. Corresponding to the glioma locations in the four subsystems of language-related areas, we categorized the patients into four subgroups, the left frontal glioma subgroup (LFG), left temporal glioma subgroup (LTG), right frontal glioma subgroup (RFG), and right temporal glioma subgroup (RTG), to study the effect of lesion topography on language network functional connectivity (LNFC). This study was approved by the ethics committee of Beijing Tiantan Hospital, and written informed consent was obtained from all participants.

All participants were assessed by an experienced surgeon (J.Y.Y.) to identify the general psychological status on the day of MRI. Specifically, we assessed cognitive capability by the Mini Mental State Examination (MMSE), and motor and mental statuses were assessed with the Karnofsky Performance Scale (KPS) (14), Beck Depression Inventory (BDI) (15).

\section{Imaging Protocols}

We acquired whole-brain fMRI data on a Siemens Prisma 3.0 Tesla scanner (Siemens Healthineers, Erlangen, Germany) with a 20-channel head coil. During the scanning, subjects were asked to relax and close their eyes. Three types of images were collected: (i) High-resolution 3D T1-weighted images; (ii) Resting-state fMR images; and (iii) T2-weighted images. The 3D T1-weighted sagittal images were acquired with a magnetism-prepared rapid acquired with gradient echo (MPRAGE) sequence: 192 slices, acquisition time $=7.4 \mathrm{~min}$; acquisition matrix $=256 \times 256$, slice thickness $/$ gap $=1 / 0 \mathrm{~mm}, \mathrm{TI} /$ $\mathrm{TR} / \mathrm{TE}=900 / 2300 / 2.3 \mathrm{~ms}$, flip angle $=8 \mathrm{deg}$, and field of view $(F O V)=256 \times 256 \mathrm{~mm}^{2}$. The rs-fMRI data were acquired using an echo-planar image sequence: 30 axial slices, acquisition matrix $=64 \times 64$, slice thickness $/$ gap $=5 / 0.5 \mathrm{~mm}$, repetition time $=2,000 \mathrm{~ms}$, echo time $=30 \mathrm{~ms}$, and FOV $=192 \times 192 \mathrm{~mm}^{2}$. The T2 images were acquired with a turbo-spin echo (TSE) sequence along the axial plane (33 axial slices; matrix size $=448 \mathrm{x}$ 406 , slice thickness/gap $=3 / 0.9 \mathrm{~mm}$, repetition time $=5,000 \mathrm{~ms}$, echo time $=105 \mathrm{~ms}$, flip angle $=150 \mathrm{deg}$, and FOV $=220 \mathrm{x}$ $199 \mathrm{~mm}^{2}$ ).

\section{Lesion Mapping}

To define the lesion feature of each patient anatomically, we first coregistered and resliced the T2 images to the native space of the averaged $3 \mathrm{D}$ images with the trilinear interpolation method in SPM12 (SPM12; Wellcome Department of Imaging Neuroscience, University College London, UK). Then, the images were normalized to the Montreal Neurological Institute (MNI) template with a spatial resolution of $1 \times 1 \times 1 \mathrm{~mm}$. The resulting images were manually traced by a senior neurosurgeon (L.J.) with MRIcron software (http://people.cas.sc.edu/rorden/ mricron.html). A volume of interest (VOI) was created for each patient. Delineation of the affected volume was based on the same criterion for all glioma patients: the entire hyperintense portion of the coregistered $3 \mathrm{D}$ T2-weighted images. A 
neurologist (T.Y.Y.) reviewed all segmentations a second time, paying special attention to the borders of the lesions and the degree of white matter disease. Then, we used the MATLAB script get_totals provided by Ridgway (www.cs.ucl.ac.uk/staff/g. ridgway/vbm/get_totals.m.) to obtain the tumor volume (TV) of every patient. Finally, patients' tumor masks of the four subgroups were separately stacked together to construct overlapping tumor images, which are displayed in Figure 1.

\section{Image Preprocessing}

Preprocessing was conducted using the DPABI toolbox (http:// rfmri.org/dpabi). The first 10 volumes were discarded to allow for saturation effects and magnetization equilibrium. Then, the remaining images were slice-timing corrected, motion corrected, aligned with the anatomical scan, normalized to the MNI space, resampled to $3.0 \mathrm{~mm}^{3}$, and spatially smoothed with a $4-\mathrm{mm}$ FWHM Gaussian kernel. The linear trend was removed for the time series of each voxel, and several nuisance signals, including Friston's 24 head motion parameter, white matter and cerebrospinal fluid signals, were regressed out. Notably, we did not regress out the global signal because this procedure remains controversial. Finally, temporal bandpass filtering $(0.01-0.08 \mathrm{~Hz})$ was applied to the time courses. Of the 112 patients, three were excluded for excessive head motion (exclusion criteria: $3.0 \mathrm{~mm}$ and 3.0 degrees in maximum head motion). Finally, 109 glioma patients and $42 \mathrm{HCs}$ were included, and the demographic data and clinical features of all participants are summarized in
Table 1. Statistical analyses were conducted using SPSS (IBM, CA, USA), with the alpha level set at $p<0.05$ for all tests.

\section{Group ICA}

We performed ICA using GIFT software (http://icatb. sourceforge.net/, version 1.3b) for MATLAB on all subjects. Thirty independent components (ICs) were created as a groupwide spatial map of the resting-state networks.

\section{LN Analysis}

The IC showing the largest and most significant spatial correlation with the LN in the resting-state networks (RSNs) template (http:// findlab.stanford.edu/functional_ROIs.htm) was selected by the software as the target network to study. Then, we obtained the mask of the LN consisting of eight separated cerebral clusters obtained at a threshold of T $>5.0$ and two cerebellar clusters at a threshold of $\mathrm{T}>3.0$. This map was intended to identify the peak voxel coordinates of the LN for seed placement in the seed-based FC analysis. The LN maps identified by group-level ICA were shown in Figure 2 and included the bilateral inferior frontal gyri (bi-IFG), bilateral middle frontal gyri (bi-MFG), bilateral superior temporal gyri (bi-STG), medial cingulate cortices (anterior, posterior), and bilateral cerebellar cortices.

\section{FC Analysis}

A $6 \mathrm{~mm}$ spherical region of interest (ROI) was placed at the peak voxel of each LN cluster in the MNI template space. The LN
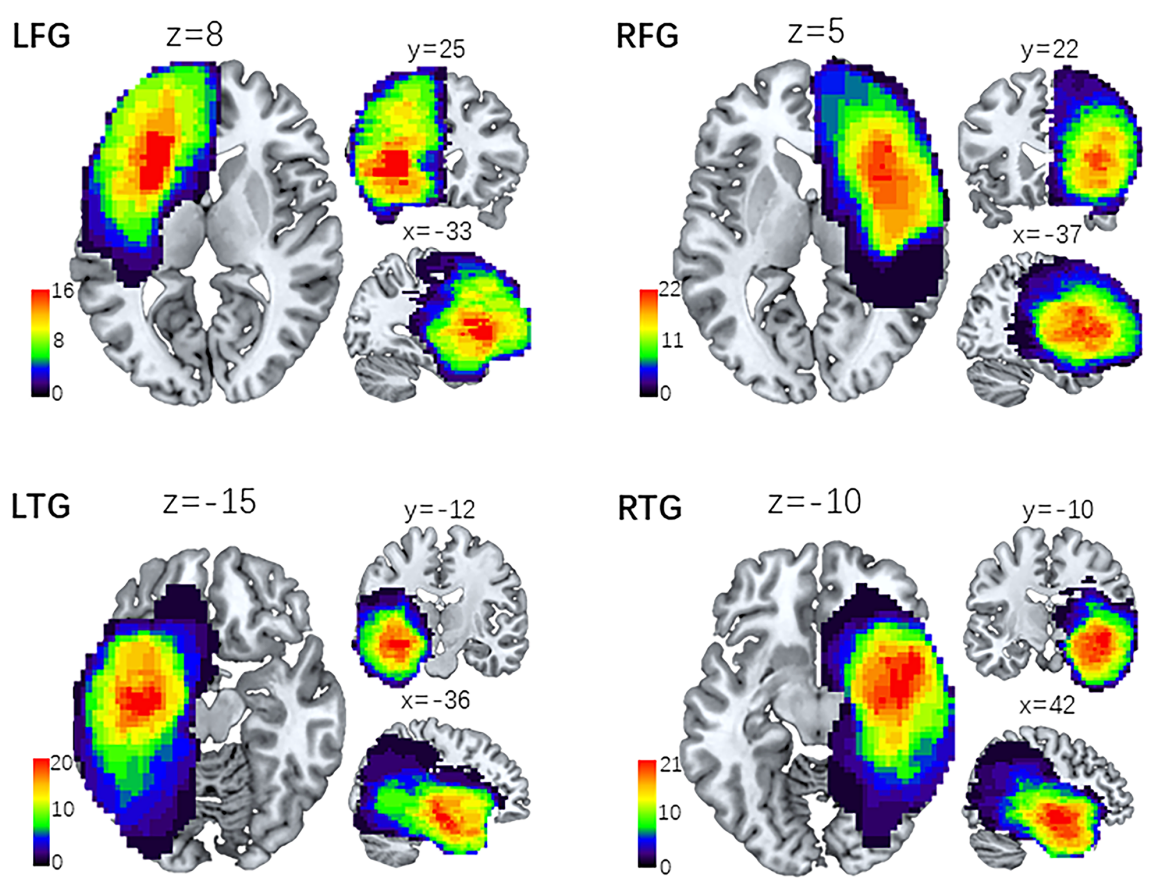

FIGURE 1 | Lesion overlap maps of four subgroups of patients. Lesion distributive density maps are obtained by stacking together the manually traced tumor volume of interest (VOI). Then, the four groups of lesion maps are overlapped onto the MNI template separately. The coordinates (x, $\mathrm{y}$, z) represent three dimensional positions on the standard template, and the color bar indicates the number of VOI in a given voxel. LFG, left frontal glioma; LTG, left temporal glioma; RFG, right frontal glioma; RTG, right temporal glioma. 
TABLE 1 | Sample demographic and clinical characteristics.

\begin{tabular}{|c|c|c|c|c|c|c|c|}
\hline Variable & LFG(27) & LTG(26) & RFG(29) & RTG(27) & $\mathrm{HC}(42)$ & $\mathrm{F} / \chi^{2}$ & $P$ \\
\hline Age (mean $\pm S D, y)$ & $45.04 \pm 12.82$ & $42.35 \pm 11.53$ & $40.76 \pm 12.68$ & $43.44 \pm 13.93$ & $39.55 \pm 9.96$ & 0.842 & $0.501^{a}$ \\
\hline Gender $(\mathrm{M} / \mathrm{F})$ & $15 / 12$ & $10 / 16$ & $15 / 14$ & $18 / 9$ & $24 / 18$ & 4.524 & $0.340^{b}$ \\
\hline $\begin{array}{l}\text { Education } \\
(\text { mean } \pm \mathrm{SD}, \mathrm{y})\end{array}$ & $11.37 \pm 3.58$ & $13.12 \pm 3.99$ & $12.61 \pm 3.78$ & $11.93 \pm 4.70$ & $13.71 \pm 3.79$ & 1.776 & $0.137^{a}$ \\
\hline $\begin{array}{l}\text { Tumor volume } \\
\left(\text { mean } \pm \mathrm{SD}, \mathrm{cm}^{3}\right)\end{array}$ & $106.6 \pm 64.60$ & $85.58 \pm 44.69$ & $110.7 \pm 67.60$ & $98.16 \pm 66.77$ & - & 0.866 & $0.461^{a}$ \\
\hline MMSE (mean \pm SD) & $28.11 \pm 1.78$ & $27.00 \pm 3.62$ & $27.41 \pm 2.73$ & $28.11 \pm 2.61$ & $29.48 \pm 1.11$ & 5.345 & $0.0005^{\mathrm{a}}$ \\
\hline Language score & $8.89 \pm 0.32$ & $8.35 \pm 1.16$ & $8.62 \pm 0.62$ & $8.56 \pm 0.80$ & $8.83 \pm 0.49$ & 2.75 & $0.03^{a}$ \\
\hline $\mathrm{BDI}($ mean $\pm \mathrm{SD})$ & $3.82 \pm 4.23$ & $2.77 \pm 4.07$ & $4.57 \pm 4.93$ & $4.11 \pm 4.36$ & $2.50 \pm 3.60$ & 1.452 & $0.220^{\mathrm{a}}$ \\
\hline KPS (mean \pm SD) & $82.59 \pm 11.30$ & $85.77 \pm 8.09$ & $81.38 \pm 11.25$ & $81.84 \pm 14.86$ & $100.0 \pm 0.00$ & 23.98 & $<0.0001^{a}$ \\
\hline \multicolumn{8}{|l|}{ Pathological types } \\
\hline Low-grade glioma & 15 & 13 & 15 & 12 & - & & \\
\hline Anaplastic astrocytoma/oligodendroglioma & 7 & 8 & 5 & 4 & - & 5.66 & $0.462^{b}$ \\
\hline Glioblastoma & 5 & 5 & 9 & 11 & - & & \\
\hline \multicolumn{8}{|l|}{ Pathological grades } \\
\hline Low-grade glioma(WHO I/II) & 15 & 13 & 15 & 12 & - & 0.692 & $0.875^{\mathrm{b}}$ \\
\hline High-grade glioma(WHO III/IV) & 12 & 13 & 14 & 15 & - & & \\
\hline
\end{tabular}

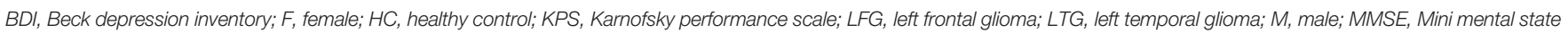

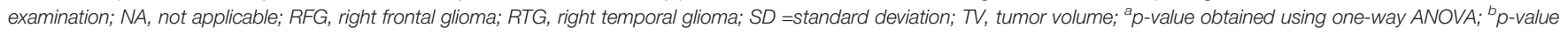
obtained using Pearson's chi-square test.
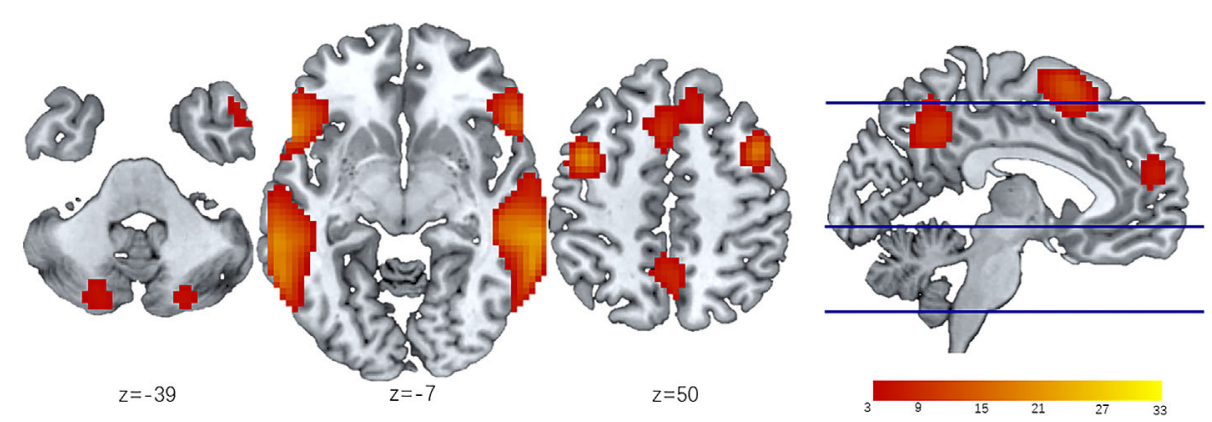

FIGURE 2 | LN identified by ICA of all participants. The LN is extracted from 30 independent components obtained from Independent component analysis (ICA). Then the group level LN map is overlap onto the MNI template to create resting state spatial map of all participants $(n=109)$. Eight separated cerebral clusters are obtained at a threshold of $\mathrm{T}>5.0$ and two cerebellar clusters are obtained at a threshold of $\mathrm{T}>3.0$. The $\mathrm{z}$-coordinates represent axial positions on the standard space, and the color bar indicates the T value of LN modules. ICA, independent component analysis; LN, language network.

ROIs and their MNI coordinates are delineated in Table 2. Mean signals extracted from the nodes were correlated with each other using Pearson's correlation coefficient (r), resulting in a total of forty-five connectivity measurements (edges). These correlation coefficients were then converted to Z-scores using Fisher's transformation. An independent two-sample $t$ test was performed to compare all edge values in each patient subgroup with those in HCs. A threshold of one-tailed false discovery rate (FDR)-corrected $p<0.05$ was applied to correct for multiple comparisons. These significantly reduced edges were classified as two different types: reduced edges connected with nodes within tumor located subsystems, which indicated connections directedly affected by tumor infiltration, and reduced edges connected between nodes in non-neoplastic modules, which suggested connections undirectedly disturbed by gliomas. Furthermore, the chi-square test was performed to analyze differences in the numbers of the two different edges between left and right hemisphere gliomas (LFG, LTG vs. RFG, RTG). $p<$ 0.05 was considered statistically significant.

\section{NC Analysis}

We selected homotopic ROIs (the same location on opposite hemispheres), including the bi-IFG, bi-MFG, and bi-STG, to balance the seed regions to obtain $\mathrm{mNC}$ representing ipsi/contralesional intrahemispheric and interhemispheric connectivity. The mNC values among the ROIs within the left and right hemispheres (intra/within-hemisphere, $1 / \mathrm{rFCw}$ ), among the ROIs between the left and right hemispheres (inter/betweenhemisphere, FCb), and among all ROIs of the LN (global, FCg) were calculated by reducing each subject's FC correlation matrix into a single variable, separately. NC analysis results were entered into one-way ANOVA. Finally, correlations between TV and mNC ( $1 / \mathrm{rFCw}, \mathrm{FCb}$, and $\mathrm{FCg})$ were assessed by using Pearson coefficients. 
TABLE 2 | List of the language network modules.

\begin{tabular}{|c|c|c|c|c|c|}
\hline \multirow[t]{2}{*}{ LN modules } & \multirow{2}{*}{$\begin{array}{l}\text { Module size } \\
\text { (in voxels) }\end{array}$} & \multicolumn{3}{|c|}{ Peak MNI Coordinates } & \multirow{2}{*}{$\begin{array}{c}\text { T score } \\
\text { peak level }\end{array}$} \\
\hline & & $x$ & $\mathbf{Y}$ & $\mathbf{Z}$ & \\
\hline$\| F G$ & 218 & -51 & 21 & -9 & 10.93 \\
\hline ISTG & 955 & -57 & -57 & 12 & 16.27 \\
\hline IMFG & 136 & -45 & 3 & 48 & 11.30 \\
\hline rIFG & 127 & 54 & 24 & -6 & 8.26 \\
\hline rSTG & 1,149 & 60 & -51 & 12 & 19.82 \\
\hline rMFG & 104 & 45 & 9 & 45 & 9.86 \\
\hline I/rSFG & 109 & -3 & 12 & 57 & 7.85 \\
\hline I/rPC & 133 & 0 & -57 & 39 & 8.39 \\
\hline ICPL & 92 & -21 & -78 & -39 & 3.47 \\
\hline rCPL & 43 & 21 & -81 & -42 & 3.17 \\
\hline
\end{tabular}

The language network modules identified by ICA. The MNI coordinates of peak voxels are listed in the table. ICA, independent component analysis; LN, language network; IIFG, left inferior frontal gyrus; ISTG, left superior temporal gyrus; IMFG, left middle frontal gyrus; I/ rSFG, left/right superior frontal gyrus; I/rPC, left/right precuneus; ICPL, left cerebellum posterior lobe; MNI, Montreal Neurological Institute. rIFG, right inferior frontal gyrus; rSTG, right superior temporal gyrus; rMFG, right middle frontal gyrus; $r C P L$, right cerebellum posterior lobe.

\section{Data Availability}

Anonymized data and data analysis pipeline will be shared by request from any qualified investigator.

\section{RESULTS}

\section{Demographic Data}

109 glioma patients and forty-two demographically matched HCs were studied (Table 1): LFG (15 males/12 females, mean age $45.04 \pm 12.82$ years); LTG (10 males/16 females, mean age $42.35 \pm 11.53$ years); RFG (15 males/14 females, mean age $40.76 \pm 12.68$ years); RTG (18 males/9 females, mean age $43.44 \pm$ 13.93 years); and HCs ( 24 males/18 females, mean age $39.55 \pm 9.96$ years). There were no significant differences among the five groups in age $[\mathrm{F}(4,146)=0.84 ; p=0.50]$, the distribution of $\operatorname{sex}\left(\chi^{2}=4.52\right.$; $p=0.34)$, or level of education $[\mathrm{F}(4,146)=1.77 ; p=0.14]$. Although language scores derived from MMSE sections demonstrated a significant difference among patient groups and $\operatorname{HCs}[\mathrm{F}(4,146)$ $=2.75 ; p=0.03]$, no remarkable difference was found among the four patient groups $[\mathrm{F}(3,105)=1.77 ; p=0.09]$. And the pathological types and grades of glioma also showed no difference $\left(\chi^{2}=5.66, p=0.462 ; \chi^{2}=0.692, p=0.875\right)$.

\section{Reduced FC Patterns in Glioma Patients}

In glioma patients, we found significantly reduced FC of LN in comparison with HCs. Figure 3 shows the distribution of FC edges with a significant reduction for the four subgroups (detailed results of the $\mathrm{T}$ values can be seen in the Supplementary material, Table S1). More importantly, we found that the left hemisphere lesions (LFG, LTG) caused much more severe disturbance to the whole-brain LN topology than the right hemisphere lesions (RFG, RTG) by comparing the numbers of reduced edges connected with nodes within tumor located subsystems (directly affected by gliomas) with edges connected between nodes in non-neoplastic modules (undirectedly disturbed by gliomas) $\left(\chi^{2}=6.399, p=0.011\right.$, Supplementary Results, Table S2).

\section{Reduced NC at the Hemisphere Level}

Having demonstrated the single-edge FC decrease in the LN in all subgroups, we further studied alterations in FC at the hemisphere level. One-way ANOVA was separately performed for $\mathrm{mNC}(1 / \mathrm{rFCw}, \mathrm{FCb}$, and $\mathrm{FCg})$ among the four subgroups of patients and HCs (Figure 4). Specifically, in terms of FCb and FCg, Tukey's test for multiple comparisons suggested significant differences between the $\mathrm{HC}$ and all four tumor subgroups (FCb: LFG $p=0.003$, LTG $p=0.001$, RFG $p<0.001$, RTG $p<0.001$, Figure 4C; FCg: LFG $p=0.005$, LTG $p<0.001$, RFG $p=0.014$, RTG $p<0.001$, Figure 4D). Interestingly, post hoc analysis confirmed that patients with left hemisphere lesions exhibited a simultaneous decrease in bilateral $\mathrm{FCw}(1 / \mathrm{rFCw})$ compared with HCs (LFG: $1 F C w ~ p=0.011, \mathrm{rFCw} p=0.014$; LTG: $\mathrm{lFCw}$ $p=0.002$, rFCw $p=0.002$, Figures 4A, B), while patients with right hemisphere lesions only exhibited a significant difference in ipsilesional FCw (rFCw) (RFG: $1 F C w p=0.760, r F C w p<0.001$; RTG: IFCw $p=0.200, \mathrm{rFCw} p=0.007$, Figures 4A, B). There was no difference in any aspect of $\mathrm{mNC}$ among the four subgroups of patients.

In addition, we did not find a remarkable difference between lFCw and $\mathrm{rFCw}$ in HCs using the paired $\mathrm{t}$ test $[\mathrm{t}(41)=0.893$, $p=0.377$, Supplementary Results, Figure S2], indicating that the strength of LNFC between the left and right hemispheres is almost equivalent under normal circumstances. Based on this result, we then explored whether there is a difference in ipsilesional versus contralesional FC (ipsi-FCw, con-FCw) using repeated measures ANOVA with $\mathrm{FCw}$ as a withinsubject factor and group as a between-subject factor. We found a main effect of $\mathrm{FCw}[\mathrm{F}(1,109)=4.98, p=0.028]$, indicating that ipsilesional FC (mean $=0.35$ ) decreased, on average, much more significantly than contralesional FC (mean $=0.41$ ) for all glioma patients. Second, when considering the $1 / \mathrm{rFCw}$ of each subgroup separately, there was a significant difference only in the RFG subgroup (paired t test: LFG $p=0.564$; LTG $p=0.743$; RFG $p=0.018$; RTG $p=0.396$; Supplementary Results, Figure S3)

\section{Association of LNFC With Glioma Topography}

Next, we investigated the extent to which lesion topography explained the decrease in FC within LN in glioma patients. For the four subgroups of patients, we separately tested the relationship between brain lesions and $\mathrm{mNC}(\mathrm{l} / \mathrm{rFCw}, \mathrm{FCb}$, and FCg) using a univariate correlation with TV. For left hemisphere lesions (LFG, LTG), TV was negatively correlated with ipsilesional (lFCw: LFG, $\mathrm{R}_{27}=-0.479, p=0.012$; LTG, $\mathrm{R}_{26}=-0.606, p=0.001$, Figures 5A, B), interhemispheric (FCb: LFG, $R_{27}=-0.510$, $p=0.007$; LTG, $R_{26}=-0.479, p=0.013$, Figures $5 E$, $\mathbf{F}$ ), and global (FCg: LFG, $\mathrm{R}_{27}=-0.482, p=0.011$; LTG, $\mathrm{R}_{26}=-0.424, p=0.031$, Figures 5E, F) FC but not with contralesional FC (rFCw: LFG, $\mathrm{R}_{27}=-0.183, p=0.360$; LTG, $\mathrm{R}_{26}=-0.206, p=0.314$, Figures 5A, B). However, in regard to the RFG and RTG subgroups, there were no remarkable negative correlations between TV and the four types of 

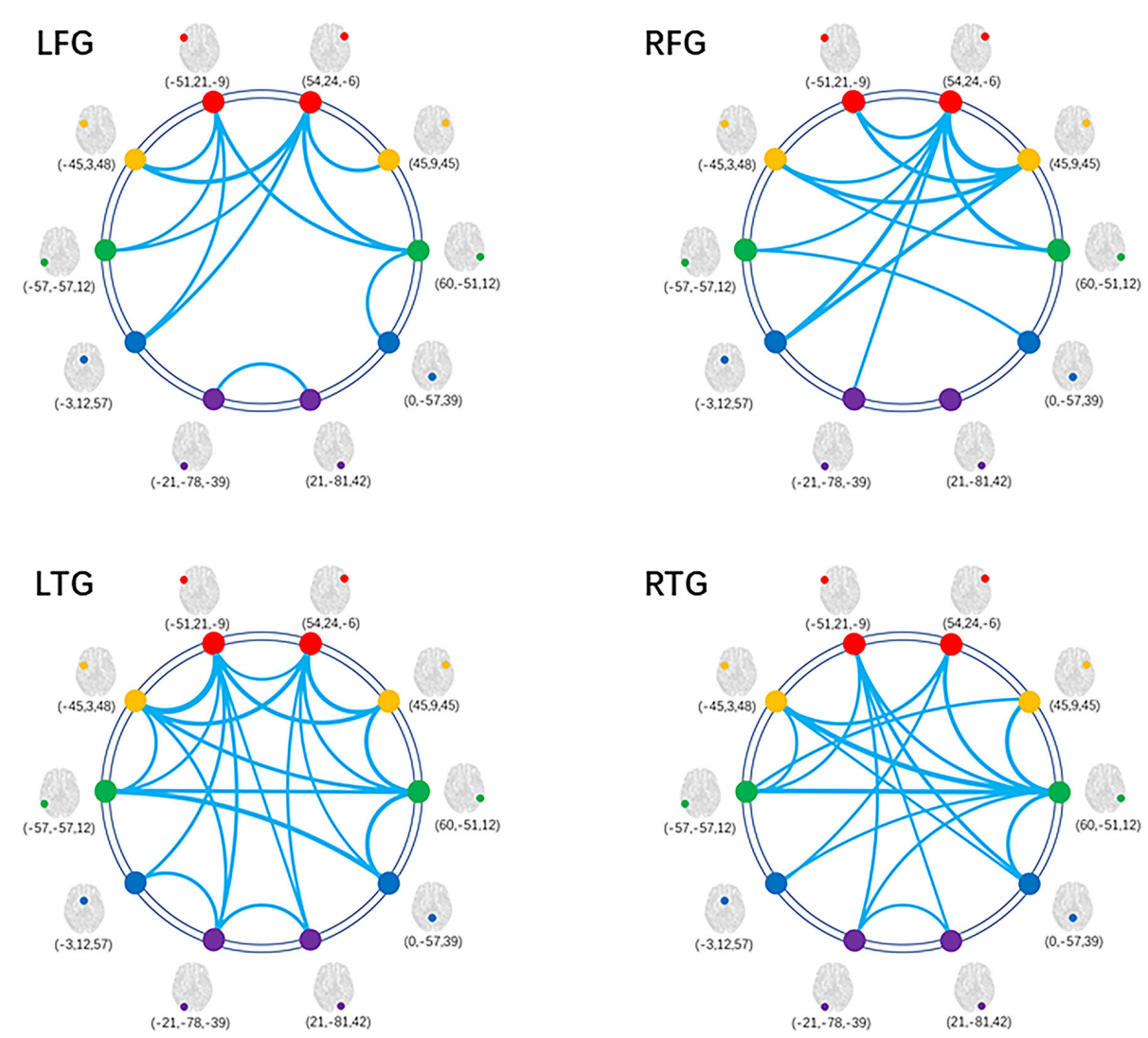

FIGURE 3 | Decreased FC within LN for patient subgroups compared with HCs. Graphical presentation of the decreased FC within LN by comparing the four subgroups of patients with $\mathrm{HCs}$ separately. The thickness of lines is proportional to the absolute value of between-group $\mathrm{T}$ (one-tailed t test, FDR-corrected $\mathrm{p}<$ 0.05). FC, functional connectivity; HC, healthy control; LN, language network; LFG, left frontal glioma; LTG, left temporal glioma; RFG, right frontal glioma; RTG, right temporal glioma.

mNC. Interestingly, we found that TV was positively correlated with contralesional intrahemispheric FC (lFCw: RFG, R29 = 0.499, $\mathrm{p}=0.006$; RTG, R27 $=0.448, \mathrm{p}=0.019$; Figures 5C, D). These contradictory results may suggest that tumors located in different language-related areas can reorganize the $\mathrm{LN}$ in different manners following the development of gliomas. And the strengths of reorganized $\mathrm{mNC}$ can be predicted by lesion volumes, but the prediction was not improved by adding information about lesion topography (pathology, grades). More details can be seen in the Supplementary material (Supplementary Results, Table S3).

\section{DISCUSSION}

In this study, we identified robust changes in LN synchrony (measured with rs-fMRI) in the setting of gliomas regardless of the specific functional-defined language areas that the tumor was involved with. However, the reduced pattern in LNFC following glioma development was modulated by tumor position. Gliomas in the left hemisphere had a broader impact on LN than gliomas in the right hemisphere, usually with weak connections between nodes located in non-neoplastic modules. Compared with HCs, $\mathrm{mNC}(1 / \mathrm{rFCw}, \mathrm{FCb}$, and $\mathrm{FCg})$ showed a significant reduction in glioma patients (except for lFCw in the right hemisphere glioma subgroups [RFG, RTG]). In addition, $\mathrm{mNC}$ has a different correlative relationship with TV depending on the tumor position across hemispheres. These results agree with the proposal that language processing is the product of dynamic interactions among domain-specific and domain-general brain regions $(16,17)$; therefore, lesions in different eloquent areas have different impacts on the LN.

It is important to note that although the gliomas of the four subgroups led to a similarly widespread decrease in LNFC, their functional profiles varied considerably. In lesion-functional topography studies, one of the key methods often used to determine the importance of an area in network communication is to examine how much the network properties change if a given area is damaged (18). Core regions, also known as lesion hubs under disease status, have the greatest contribution to the functional network and are highly indispensable in the healthy network systems. Damage to these areas can have a fatally widespread effect on the transmission of information throughout the system $(19,20)$. Our study found that left frontotemporal subsystem 
A
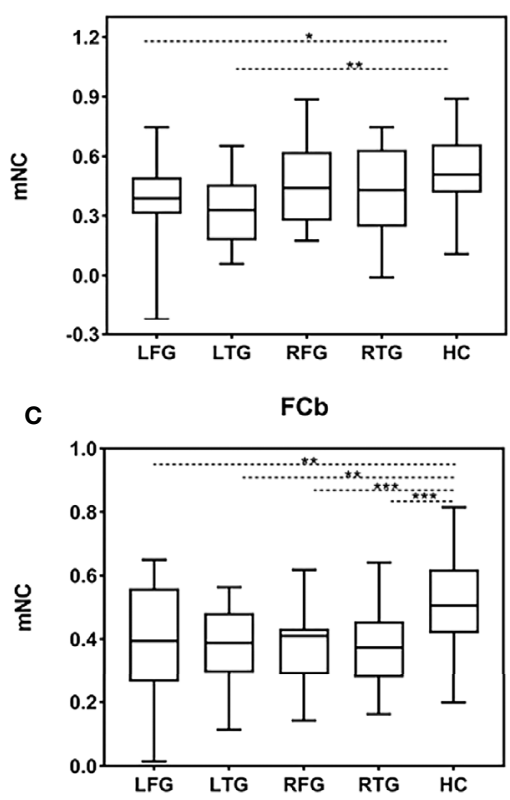

B

$\mathrm{rFCW}$
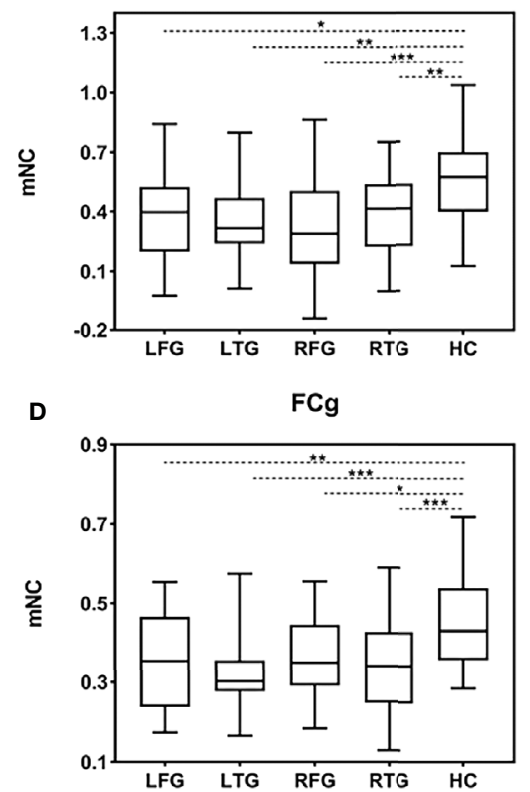

FIGURE 4 | mNC differences among the four subgroups of patients and HCs. (A-D) Group differences in mNC (//rFCw, FCb, and FCg) among the four subgroups of patients and HCs. Significant mNC differences are observed between the four subgroups of patients and HCs except for IFCW in the groups of RFG and RTG. Post hoc analysis shows that there is no difference in any aspect of $\mathrm{mNC}$ among the four subgroups of patients. Error bars represent one standard error of the mean (SEM). $\left({ }^{\star} p<0.05 ;{ }^{\star \star} p<0.01 ;{ }^{\star \star \star} p<0.001\right)$. FCb, inter/between-hemisphere functional connectivity; FCg, global functional connectivity; HC, healthy control; LFG, left frontal glioma; LTG, left temporal glioma; IFCW, left intra/within-hemisphere functional connectivity; mNC, mean network connectivity; rFCw, right intra/withinhemisphere functional connectivity; RFG, right frontal glioma; RTG, right temporal glioma.

gliomas strongly affected global LN communication, with the overwhelming majority of reduced edges away from the lesion sites, indicating that the transmission of information among remote modules in LN has been significantly disturbed. However, for right frontotemporal subsystem gliomas, most of the affected edges were connected with lesion sites, highlighting that disturbance to the right frontotemporal subsystems induced limited impacts on the whole network topology. With voxel-based lesion symptom mapping in mind, our study supports the hypothesis that both left frontal and temporal subsystems are the "core" regions of the LN; on the other hand, right frontal and temporal subsystems are the "periphery" regions $(16,17,21)$.

From a core-periphery linguistic point of view, inter- and intrahemispheric FC was studied to identify specific alterations in $\mathrm{mNC}$ in the presence of gliomas. We found that the LFG and LTG subgroups demonstrated a consistent pattern in which $\mathrm{mNC}(1 / \mathrm{rFCw}, \mathrm{FCb}$, and $\mathrm{FCg})$ decreased significantly in comparison with HCs. Moreover, decreased $\mathrm{mNC}$ between the two subgroups suggested no significant differences among the four types of mNC. By contrast, patients with right hemispheric gliomas (RFG, RTG) did not exhibit a remarkable decrease in $\mathrm{mNC}$ in the left frontotemporal subnetwork (lFCw) compared with HCs. Thus, our subsequent hemisphere level studies suggested that the LN was implemented in the model of leftdominant organization, damage to which has a very large disturbance on the LN, not only in communication among the left frontotemporal subnetwork but also in the structurally intact right frontotemporal subnetwork that participates in language processing (22). Furthermore, given the different alterations of left frontotemporal subnetwork, $\mathrm{lFCw}$ may be considered a sensitive indicator for distinguishing the extent of LNFC reorganization in the context of gliomas before surgery.

Most previous studies have revealed that the left frontotemporal network is crucial and specialized for language functions $(23,24)$. Comprehension and repetition deficits are associated with left frontotemporal brain damage, and lesion-deficit studies have revealed that lesion volume and the fractional anisotropy (FA) value of subcortical tissue are significantly correlated with the severity of impairment in language behavior $(25,26)$. Furthermore, the right hemisphere contributes to linguistic processing and working memory, which are needed for normal speech comprehension dynamically (27). Pathologies on the right temporal and frontal cortex tend to have a selective effect on language performance (e.g., cognitive control, working memory, executive function) $(7,28)$. However, our combined bilateral glioma study confirmed that right hemisphere structural damage only diminished subnetwork level connections among the periphery $(\mathrm{rFCw})$ rather than core modules (lFCw). Thus, some aspects of key language functions may still be preserved in right hemisphere glioma patients; however, their language systems could be less synchronized, diminishing their ability to adapt dynamically to language processing demands (29). 
A

LFG

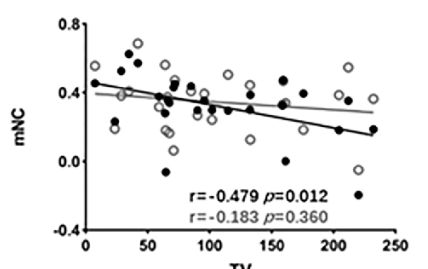

TV

E

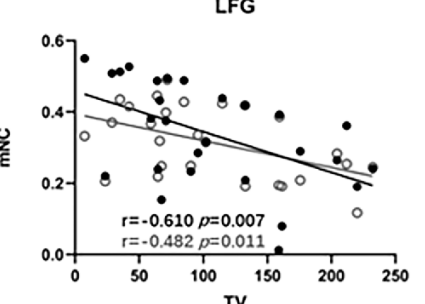

$$
\rightarrow \mathrm{IFCW}
$$

LTG

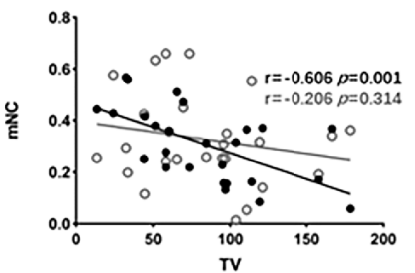

F

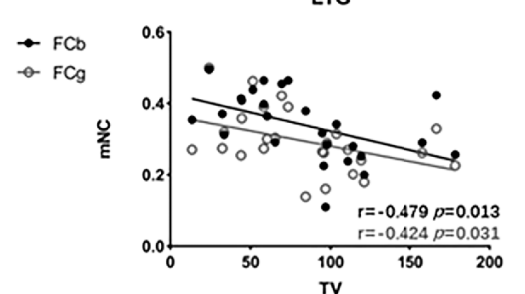

C

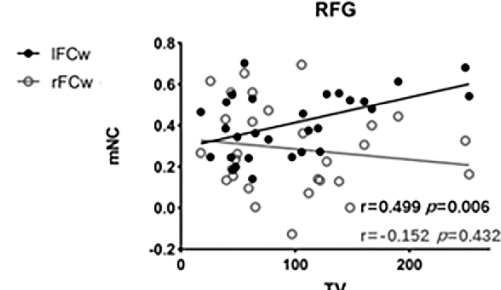

TV

G

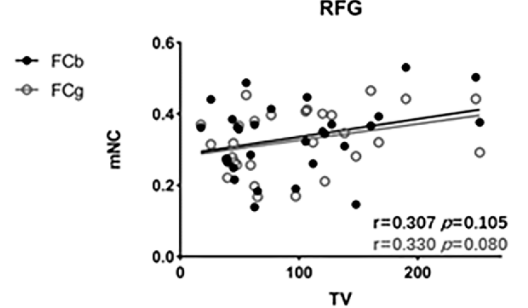

D

$\rightarrow$ IFCW

$\rightarrow$ rFCW

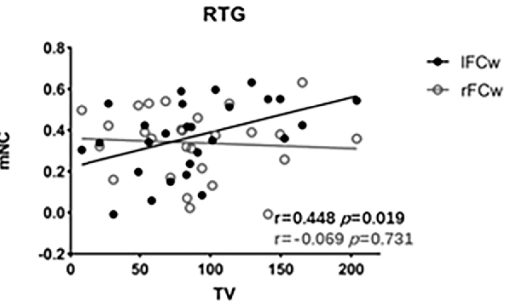

H

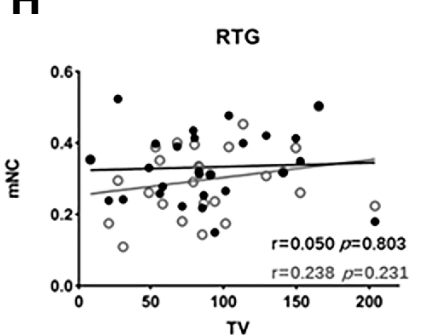

FIGURE 5 | Associations between $m N C$ and TV. Associations between $m N C$ ( $\left(/ / F C W, F C b\right.$, and FCg) and lesion volume. For $L F G$ and $L T G$, TV is negatively correlated with ipsilesional (IFCW: LFG, $R_{27}=-0.479$, $p=0.012$; $L T G, R_{26}=-0.606, p=0.001$, A, B), interhemispheric ( $\left.F C b: L F G, R_{27}=-0.510, p=0.007 ; \quad L T G, R_{26}=-0.479, p=0.013, E, F\right)$, and global (FCg: $L F G, R_{27}=-0.482, p=0.011 ; L T G, R_{26}=-0.424, p=0.031,(\mathbf{E}, \mathbf{F})$; while, for the RFG and RTG, positive correlations are found between IFCW and TV (IFCW: RFG, $R_{29}=0.499, p=0.006$; RTG, $R_{27}=0.448, p=0.019$; (C, D) and there are no remarkable negative correlations between TV and the other types of $\mathrm{mNC}$ (rFCw: RFG, R29 = -0.152, $p=0.432$, RTG, R27 = -0.069, $p=0.731, \mathrm{C}, \mathrm{D}$; FCb: RFG, R29 = 0.307, $p=0.105, R T G, R 27=0.050, p=0.803 ; F C g: R F G, R 29=0.330, p=0.080, R T G$, $R 27=0.238, p=0.231, \mathbf{G}, \mathbf{H})$. FCb, inter/between-hemisphere functional connectivity; FCg, global functional connectivity; IFCw, left intra/within-hemisphere functional connectivity; LFG, left frontal glioma; LTG, left temporal glioma; mNC, mean network connectivity; rFCW, right intra/within-hemisphere functional connectivity; RFG, right frontal glioma; RTG, right temporal glioma; TV, tumor volume. 
The neurological mechanism underlying FC reduction between LN modules following glioma remains unclear. A possible explanation is the neurovascular uncoupling (NVU) -the abnormal tumor neo-vasculature may arouse disturbances over neuronal metabolism and neurotransmitter, which would affect the consistency and synchrony of neural functional activities at the whole brain level (30-32). In line with this notion, our findings additionally highlighted that the disturbances of connectivity with LN were associated with pathological topographies, especially lesion volumes and sites. For left hemisphere lesions (LFG, LTG), mNC, including ipsilesional intrahemispheric (lFCw), interhemispheric (FCb), and global (FCg) FC, was negatively correlated with TV. However, for right hemisphere lesions (RFG, RTG), there were no negative correlations between TV and the four types of mNC. Notably, although right hemispheric gliomas did not exhibit a significant decrease in contralesional intrahemispheric FC (lFCw) compared with $\mathrm{HCs}$, a positive correlation was demonstrated between TV and IFCw. These interesting results suggest that disrupted communication among modules of LN is a central feature of left hemisphere lesions. Conversely, for right hemisphere lesions, it was likely to exist a dynamic functional compensation among LN core modules associated with tumor progression, which reflect the reconfiguration mechanism of core-periphery subsystems to regions most capable of meeting linguistic operations.

Our study found that the human language system retains functional organization under a hierarchical network framework, the processing of which depends on bilateral frontotemporal language areas. Language deficits can arise not only from a pure disruption in functionally specialized cores but also from a disturbance of communication among the functionally interconnected remote modules within LN (33). As demonstrated by numerous functional imaging studies, language disorders are well predicted both by lesion topography and FC: the former's primary principle is that specific language functions dominate in specific regions of the brain, and the latter is based on the theory that highlevel cognitive function is embedded with the connections among a large-scale functional network. Importantly, in addition to lesionbehavior and FC-behavior mapping, our study provides a more fundamental association between lesion topography and LNFC on the basis of cognitive functional studies. Given recent evidence for network data to provide prognostic markers (34-36), particularly in relation to postsurgical language outcomes, it is therefore anticipated that a combination of core-periphery connectome models with brain lesion topography will further our understanding of associated language dysfunctions and the likelihood of recovery following an operation-a crucial factor in facilitating surgical strategies and improving the care of glioma patients.

\section{CONCLUSIONS}

Our findings emphasize the importance of considering the modulatory effects of core-periphery mechanisms when considering language reconfiguration for glioma patients from a network perspective. Lesion-behavior and FC-behavior models are important predictors for language dysfunction. The correlative relationship of these two factors suggests that a much broader approach is needed to evaluate language functional reorganization following glioma development. Recent evidence indicates that language recovery after surgery in glioma patients is linked to the organization patterns of the presurgical language system (37). Therefore, our findings of LN core-periphery organizational forms may provide the surgeon a reference to optimize resection of gliomas while maintaining a functional balance as well as a unique opportunity to further our understanding of the language system.

\section{DATA AVAILABILITY STATEMENT}

The raw data supporting the conclusions of this article will be made available by the authors, without undue reservation.

\section{ETHICS STATEMENT}

The studies involving human participants were reviewed and approved by the Ethics Committee of Beijing Tiantan Hospital. The patients/participants provided their written informed consent to participate in this study.

\section{AUTHOR CONTRIBUTIONS}

LJ: design and conceptualized study, analyzed the data, and drafted the manuscript for intellectual content. CL: design and conceptualized study. YZ: design and conceptualized study. TY: major role in the acquisition of data. JY: major role in the acquisition of data. ZZ: interpreted the data and revised the manuscript for intellectual content. SG: interpreted the data and revised the manuscript for intellectual content. All authors contributed to the article and approved the submitted version.

\section{FUNDING}

Our study is funded by Beijing Municipal Science \& Technology Commission (Grant No. Z171100000117002), Beijing Municipal Science \& Technology Commission (Grant No. Z191100006619087), and National Natural Science Foundation of China (Nos. 61872020).

\section{SUPPLEMENTARY MATERIAL}

The Supplementary Material for this article can be found online at: https://www.frontiersin.org/articles/10.3389/fonc.2021. 617179/full\#supplementary-material 


\section{REFERENCES}

1. Dronkers NF, Wilkins DP, Van Valin RD, Redfern BB, Jaeger JJ. Lesion analysis of the brain areas involved in language comprehension. Cognition (2004) 92:145-77. doi: 10.1016/j.cognition.2003.11.002

2. Just MA, Carpenter PA, Keller TA, Eddy WF, Thulborn KR. Brain activation modulated by sentence comprehension. Science (New York N Y) (1996) 274:114-6. doi: 10.1126/science.274.5284.114

3. Duffau $\mathrm{H}$. The huge plastic potential of adult brain and the role of connectomics: new insights provided by serial mappings in glioma surgery. Cortex J Devoted Study Nerv Syst Behav (2014) 58:325-37. doi: 10.1016/ j.cortex.2013.08.005

4. Cho NS, Peck KK, Zhang Z, Holodny AI. Paradoxical Activation in the Cerebellum During Language fMRI in Patients with Brain Tumors: Possible Explanations Based on Neurovascular Uncoupling and Functional Reorganization. Cerebellum (Lond Engl) (2018) 17:286-93. doi: 10.1007/ s12311-017-0902-5

5. Campbell KL, Tyler LK. Language-related domain-specific and domaingeneral systems in the human brain. Curr Opin Behav Sci (2018) 21:132-7. doi: 10.1016/j.cobeha.2018.04.008

6. Sollmann N, Tanigawa N, Ringel F, Zimmer C, Meyer B, Krieg SM. Language and its right-hemispheric distribution in healthy brains: an investigation by repetitive transcranial magnetic stimulation. NeuroImage Null (2014) 70:18595. doi: 10.1016/j.neuroimage.2014.09.002

7. Gajardo-Vidal A, Lorca-Puls DL, Hope TMH, Parker Jones O, Seghier ML, Prejawa S, et al. How right hemisphere damage after stroke can impair speech comprehension. Brain: J Neurol (2018) 141:3389-404. doi: 10.1093/brain/ awy 270

8. Hagoort P. The core and beyond in the language-ready brain. Neurosci Biobehav Rev (2017) 81:194-204. doi: 10.1016/j.neubiorev.2017.01.048

9. Fedorenko E, Behr MK, Kanwisher N. Functional specificity for high-level linguistic processing in the human brain. Proc Natl Acad Sci U S A (2011) 108:16428-33. doi: 10.1073/pnas.1112937108

10. Zhang N, Xia M, Qiu T, Wang X, Lin CP, Guo Q, et al. Reorganization of cerebro-cerebellar circuit in patients with left hemispheric gliomas involving language network: A combined structural and resting-state functional MRI study. Hum Brain Mapp (2018) 39:4802-19. doi: 10.1002/hbm.24324

11. Briganti C, Sestieri C, Mattei PA, Esposito R, Galzio RJ, Tartaro A, et al. Reorganization of functional connectivity of the language network in patients with brain gliomas. AJNR Am J Neuroradiol (2012) 33:1983-90. doi: 10.3174/ ajnr.A3064

12. He X, Bassett DS, Chaitanya G, Sperling MR, Kozlowski L, Tracy JI. Disrupted dynamic network reconfiguration of the language system in temporal lobe epilepsy. Brain: J Neurol (2018) 141:1375-89. doi: 10.1093/brain/awy042

13. Oldfield RC. The assessment and analysis of handedness: the Edinburgh inventory. Neuropsychologia (1971) 9:97-113. doi: 10.1016/0028-3932(71) 90067-4

14. Clancey JK. Karnofsky performance scale. J Neurosci Nurs J Am Assoc Neurosci Nurses (1995) 27:220.

15. Reiland SA. Beck Depression Inventory. In: V Zeigler-Hill, TK Shackelford, editors. Encyclopedia of Personality and Individual Differences. Cham: Springer International Publishing (2017). p. 1-3. doi: 10.1007/978-3-31928099-8_6-1

16. Rodd JM, Johnsrude IS, Davis MH. The role of domain-general frontal systems in language comprehension: evidence from dual-task interference and semantic ambiguity. Brain Lang (2010) 115:182-8. doi: 10.1016/ j.bandl.2010.07.005

17. Klimovich-Gray A, Bozic M. Domain-general and domain-specific computations in single word processing. NeuroImage Undefined (2019) 202:116112. doi: 10.1016/j.neuroimage.2019.116112

18. Gratton C, Nomura EM, Pérez F, D'Esposito M. Focal brain lesions to critical locations cause widespread disruption of the modular organization of the brain. J Cogn Neurosci (2012) 24:1275-85. doi: 10.1162/jocn_a_00222

19. Honey CJ, Sporns O. Dynamical consequences of lesions in cortical networks. Hum Brain Mapp (2008) 29:802-9. doi: 10.1002/hbm.20579

20. Aerts H, Fias W, Caeyenberghs K, Marinazzo D. Brain networks under attack: robustness properties and the impact of lesions. Brain J Neurol (2016) 139:3063-83. doi: 10.1093/brain/aww194
21. Kiran S, Thompson CK. Neuroplasticity of Language Networks in Aphasia: Advances, Updates, and Future Challenges. Front Neurol (2019) 10:295. doi: 10.3389/fneur.2019.00295

22. Hickok G, Poeppel D. The cortical organization of speech processing. Nat Rev Neurosci (2007) 8:393-402. doi: 10.1038/nrn2113

23. Yang M, Li Y, Li J, Yao D, Liao W, Chen H. Beyond the Arcuate Fasciculus: Damage to Ventral and Dorsal Language Pathways in Aphasia. Brain Topogr (2017) 30:249-56. doi: 10.1007/s10548-016-0503-5

24. Rosso C, Vargas P, Valabregue R, Arbizu C, Henry-Amar F, Leger A, et al. Aphasia severity in chronic stroke patients: a combined disconnection in the dorsal and ventral language pathways. Neurorehabil Neural Repair (2015) 29:287-95. doi: 10.1177/1545968314543926

25. Han Z, Ma Y, Gong G, He Y, Caramazza A, Bi Y. White matter structural connectivity underlying semantic processing: evidence from brain damaged patients. Brain: J Neurol (2013) 136:2952-65. doi: 10.1093/ brain/awt205

26. Kümmerer D, Hartwigsen G, Kellmeyer P, Glauche V, Mader I, Klöppel S, et al. Damage to ventral and dorsal language pathways in acute aphasia. Brain: J Neurol (2013) 136:619-29. doi: 10.1093/brain/aws354

27. Yang $\mathrm{X}$, Zhang $\mathrm{X}$, Yang $\mathrm{Y}$, Lin N. How context features modulate the involvement of the working memory system during discourse comprehension. Neuropsychologia (2018) 111:36-44. doi: 10.1016/j.neuropsychologia. 2018.01.010

28. Dietrich S, Hertrich I, Seibold VC, Rolke B. Discourse management during speech perception: A functional magnetic resonance imaging (fMRI) study. NeuroImage (2019) 202:116047. doi: 10.1016/j.neuroimage.2019.116047

29. Fedorenko E, Thompson-Schill SL. Reworking the language network. Trends Cogn Sci (2014) 18:120-6. doi: 10.1016/j.tics.2013.12.006

30. Fraga de Abreu VH, Peck KK, Petrovich-Brennan NM, Woo KM, Holodny AI. Brain Tumors: The Influence of Tumor Type and Routine MR Imaging Characteristics at BOLD Functional MR Imaging in the Primary Motor Gyrus. Radiology (2016) 281:876-83. doi: 10.1148/radiol.2016151951

31. Hou BL, Bradbury M, Peck KK, Petrovich NM, Gutin PH, Holodny AI. Effect of brain tumor neovasculature defined by rCBV on BOLD fMRI activation volume in the primary motor cortex. NeuroImage (2006) 32:489-97. doi: 10.1016/j.neuroimage.2006.04.188

32. Holodny AI, Schulder M, Liu WC, Wolko J, Maldjian JA, Kalnin AJ. The effect of brain tumors on BOLD functional MR imaging activation in the adjacent motor cortex: implications for image-guided neurosurgery. AJNR Am J Neuroradiol (2000) 21:1415-22.

33. Abel S, Weiller C, Huber W, Willmes K, Specht K. Therapy-induced brain reorganization patterns in aphasia. Brain: J Neurol (2015) 138:1097-112. doi: 10.1093/brain/awv022

34. Geranmayeh F, Leech R, Wise RJS. Network dysfunction predicts speech production after left hemisphere stroke. Neurology (2016) 86:1296-305. doi: 10.1212/WNL.0000000000002537

35. Siegel JS, Ramsey LE, Snyder AZ, Metcalf NV, Chacko RV, Weinberger K, et al. Disruptions of network connectivity predict impairment in multiple behavioral domains after stroke. Proc Natl Acad Sci U S A (2016) 113:E436776. doi: $10.1073 /$ pnas. 1521083113

36. Herbet G, Maheu M, Costi E, Lafargue G, Duffau H. Mapping neuroplastic potential in brain-damaged patients. Brain: J Neurol (2016) 139:829-44. doi: 10.1093/brain/awv394

37. Kristo G, Raemaekers M, Rutten GJ, de Gelder B, Ramsey NF. Interhemispheric language functional reorganization in low-grade glioma patients after tumour surgery. Cortex J Devoted Study Nerv Syst Behav (2015) 64:235-48. doi: 10.1016/j.cortex.2014.11.002

Conflict of Interest: The authors declare that the research was conducted in the absence of any commercial or financial relationships that could be construed as a potential conflict of interest.

Copyright $\odot 2021 \mathrm{Jin}$, Li, Zhang, Yuan, Ying, Zuo and Gui. This is an open-access article distributed under the terms of the Creative Commons Attribution License (CC BY). The use, distribution or reproduction in other forums is permitted, provided the original author(s) and the copyright owner(s) are credited and that the original publication in this journal is cited, in accordance with accepted academic practice. No use, distribution or reproduction is permitted which does not comply with these terms. 